\title{
Units in some parametric families of quartic fields
}

\author{
by \\ Franck Leprévost (Luxembourg), Michael Pohst (Berlin) \\ and ANDREAS SchöpP (Berlin)
}

1. Introduction. Let $F$ be a number field generated by a zero $\varrho$ of a monic irreducible polynomial $f \in \mathbb{Z}[x]$. Let $n_{F}$ be the degree of $F$ and $r_{F}$ the unit rank of $F$. The computation of the unit group of an order of $F$ can be done by several methods like the Voronoi algorithm $\left(r_{F} \leq 2\right)$, successive minima and other geometric methods using parallelotopes and ellipsoids. If $f$ defines a parametric family of polynomials it is a problem to give a fundamental system of units of $F$ in a parametric form, in particular for increasing degree $n_{F}$ and rank $r_{F}$.

In this article we only consider parametric families of quartic fields. In the case $n_{F}=4$ Stender ([16], [17]) has obtained families with unit rank 2 . Some families with unit rank 3 are described in the biquadratic case ([15], [1], [3], [18]). In the non-biquadratic case such families have been published in several articles, for example Washington ([19]), Lecacheux ([5], [4]), Lettl and Pethö ([7]), Nakamula ([10]) and Niklasch and Smart ([11]). These families are different from the three presented here: In [19] and [7] cyclic number fields are studied, and the families in [5] are also abelian with Galois group $C_{4}$ or $V_{4}$. The polynomials in [11] have Galois group $S_{4}$, and the generated number fields have unit rank 2 while in [4] the generating polynomials have Galois group $D_{4}$, and the generated number fields are totally real with unit rank 3. In [10] there are three parametric polynomials with Galois group $D_{4}$ considered: one family of number fields with unit rank 1, one with unit rank 2 and the last has unit rank 3 . The second family generates, for almost all choices of the parameter, number fields with signature $(2,1)$, but the polynomials with Galois group $D_{4}$ of our first family have for different choices of the parameter infinitely often signature $(2,1)$ and $(4,0)$. The other two families presented here have Galois group $S_{4}$ and signature $(4,0)$.

2000 Mathematics Subject Classification: 11R27, 11R16, 11R32.

Key words and phrases: cyclic Galois group, dihedral Galois group, fundamental units. 
In Section 2 of this article we compute parametric units for a family of number fields presented in [6]. There we have constructed polynomials $F_{n}(x)$ of degree $n$ by using elliptic curves with rational points of order $n$. The polynomials have Galois group either the dihedral group $D_{n}$ of order $2 n$, or the cyclic group $C_{n}$ of order $n$. Here we consider the case $n_{F}=4$, and we compute parametric units which form a fundamental system of units under some conditions. In [14] the case $n_{F}=5$ is examined.

In the last two sections, we present two new families of totally real quartic number fields and compute parametric fundamental systems of units. The first family arises from the same idea as the families in [10] but is not included there.

2. Family with Galois group $D_{4}$ or $C_{4}$. For $n \in \mathbb{Z}$ we consider polynomials

$$
F_{b}(x):=x^{4}-n x^{3}+b(n-1) x^{2}+2 b^{2} x-b^{3} .
$$

These polynomials were already considered in [6] for other purposes. They have discriminants

$$
d_{b}=d\left(F_{b}\right)=(4(n-4 b)+1)\left(n^{2}+4 b\right)^{2} .
$$

To compute parametric units of the number fields $F$ generated by $F_{b}$ we consider only $b= \pm 1$. Furthermore we assume from now on that $(b, n) \in$ $\{(-1, \pm 2),(1,0),(1,4)\}$, hence the polynomials $F_{b}$ are irreducible.

Theorem 2.1. The polynomial $F_{1}$ has signature $(2,1)$ for $n \leq 3$ and $(4,0)$ for $n \geq 4$. The polynomial $F_{-1}$ has signature $(2,1)$ for $n \leq-5,(0,2)$ for $n \in\{-4,-3,-1,0,1\}$ and $(4,0)$ for $n \geq 3$.

For $n \leq 3$ the discriminant $d_{1}$ is negative, for $n \geq 4$ it is positive. Because of $F_{1}(0)=-1$ the polynomial $F_{1}$ has at least one real zero, hence all zeros are real.

The discriminant $d_{-1}$ is negative for $n \leq-5$, and positive for $n \geq-4$. For $n \geq 3$ we have $F_{-1}(1)=1-n+(1-n)+2+1=5-2 n<0$ so that $F_{-1}$ again has at least one and therefore four real zeros. In the remaining cases $n \in\{-4,-3,-1,-0,1\}$ one easily checks that the signature is $(0,2)$.

We want the polynomials $F_{b}$ to generate quartic fields containing exactly one quadratic subfield. A candidate for the discriminant of (an order of) such a quadratic field is clearly $n^{2} \pm 4$. Therefore we make

First Assumption: $n^{2}+4 b$ is not a square. Clearly, this is tantamount to $(n, b) \neq(0,1)$.

TheOREM 2.2. $\Omega_{b}:=\mathbb{Q}\left(\sqrt{n^{2}+4 b}\right)$ is a quadratic number field. The polynomial $F_{b}$ splits over this field as

$$
F_{b}(x)=\left(x^{2}+\varepsilon x-\varepsilon b\right)\left(x^{2}+\bar{\varepsilon} x-\bar{\varepsilon} b\right)
$$


with a unit $\varepsilon=\frac{1}{2}\left(-n+\sqrt{n^{2}+4 b}\right) \in \Omega_{b}$ of norm $-b$. (The bar denotes the non-trivial automorphism of the quadratic field.)

The proof is by a straightforward calculation.

REMARK. It is well known [9] that $n^{2} \pm 4$ is square-free for infinitely many $n \in \mathbb{Z}$, hence $\varepsilon$ is the fundamental unit of $\Omega_{b}$ in those cases, except for $n=3, b=-1$, where $\varepsilon$ is the cube of the fundamental unit.

REMARK. If $F_{b}$ is irreducible with Galois group $V_{4}$ then $4(n-4 b)+1$ is a square.

THEOREM 2.3. If $4(n-4 b)+1$ is not a square in $\mathbb{Z}$ then the polynomial $F_{b}$ has Galois group $D_{4}$ or $C_{4}$.

The polynomial $F_{b}$ is irreducible over $\mathbb{Q}$ if and only if the polynomial

$$
x^{2}+\varepsilon x-\varepsilon b
$$

is irreducible in $\Omega_{b}[x]$. That polynomial is reducible if and only if $\alpha:=$ $\varepsilon^{2}+4 \varepsilon b$ is a square in $\Omega_{b}$. But in that case $N(\alpha)=N(\varepsilon(\varepsilon+4 b))=4 n+1-16 b$ is a square in $\mathbb{Q}$, which contradicts our premises. Together with the preceding remark we obtain the theorem.

We note that $4 n+1-16 b$ is a square if and only if $n=u^{2}+u+4 b$ for some $u \in \mathbb{Z}$.

Because of Theorem 2.3 and because we want to have Galois group $D_{4}$ or $C_{4}$ we make

Second Assumption: $4(n-4 b)+1$ is not a square in $\mathbb{Z}$.

TheOREM 2.4. The polynomial $F_{b}$ generates a Galois extension over $\mathbb{Q}$ (with Galois group $C_{4}$ ) if and only if for $\alpha:=\varepsilon^{2}+4 \varepsilon b$ the quotient $\alpha / \bar{\alpha}$ is a square in $\Omega_{b}$. The latter is tantamount to $4(n-4 b)+1$ being a square in $\Omega_{b}$.

At this stage we know that a root $\varrho$ of $F_{b}$ generates a quartic extension of $\mathbb{Q}$. Hence, the square-roots of $\alpha=\varepsilon^{2}+4 \varepsilon b$ and of $\bar{\alpha}$ generate quadratic extensions of $\Omega_{b}$. If and only if these extensions coincide, either of them will be a cyclic extension of $\mathbb{Q}$. In that case, we have $\sqrt{\alpha}=\mu+\nu \sqrt{\bar{\alpha}}$ with some $\mu, \nu \in \Omega_{b}$. Squaring this equation leads to $\mu \nu=0$, hence $\mu=0$. Therefore $\alpha / \bar{\alpha}$ must be a square in $\Omega_{b}$. Because of

$$
\frac{\alpha}{\bar{\alpha}}=\frac{N(\alpha)}{(\bar{\alpha})^{2}}
$$

and $N(\alpha)=4 n+1-16 b$ the theorem follows.

As mentioned in Theorem 2.4 the polynomial $F_{b}$ has Galois group $C_{4}$ if and only if $4 n+1-16 b$ is a square in $\Omega_{b}$. The latter is tantamount to $v^{2}(1+4 n-16 b)=n^{2}+4 b$ with $n, v \in \mathbb{Q}$. 
Theorem 2.5. The polynomial $F_{b}$ generates a Galois extension over $\mathbb{Q}$ with Galois group $C_{4}$ only for $(b, n) \in\{(1,8),(-1,-3),(-1,7)\}$.

To prove this we first consider $b=1$. That means we want to solve $v^{2}(4 n-15)=n^{2}+4$, which implies $n_{1 / 2}=2 v^{2} \pm \sqrt{4 v^{4}-15 v^{2}-4}$. We have $n \in \mathbb{Q}$ if $4 v^{4}-15 v^{2}-4$ is a square in $\mathbb{Q}$, in other words if the elliptic curve $E_{1}$ of equation $y^{2}=4 v^{4}-15 v^{2}-4$ has at least one rational point $(v, y) \in \mathbb{Q}^{2}$.

The Weierstraß form of $E_{1}$ is

$$
z^{2}=t^{3}-11 t-890
$$

Computations with the computer algebra system Magma [8] show that $E_{1}(\mathbb{Q}) \simeq \mathbb{Z} / 4 \mathbb{Z}=\left\{\mathcal{O}, P_{1}, P_{2}, P_{3}\right\}$, with

\begin{tabular}{ccccc}
\hline & $z$ & $t$ & $y$ & $v$ \\
\hline$P_{1}$ & 136 & 27 & $\infty$ & $\infty$ \\
$P_{2}$ & 0 & 10 & 0 & -2 \\
$P_{3}$ & -136 & 27 & $\infty$ & $\infty$ \\
\hline
\end{tabular}

That means in the case $b=1$ we get the Galois group $C_{4}$ only for $n=8$ corresponding to the polynomial $x^{4}-8 x^{3}+7 x^{2}+2 x-1$.

For the second case, $b=-1$, the same considerations show that $n_{1 / 2}=$ $2 v^{2} \pm \sqrt{4 v^{4}+17 v^{2}+4}$ has to be a rational number, which implies the existence of rational points on the elliptic curve $E_{-1}$ of Weierstraß equation

$$
z^{2}=t^{3}-12987 t-263466 .
$$

Computations show that $E_{-1}(\mathbb{Q}) \simeq \mathbb{Z} / 8 \mathbb{Z}=\left\{\mathcal{O}, P_{1}, P_{2}, \ldots, P_{7}\right\}$, where

\begin{tabular}{ccccc}
\hline & $t$ & $z$ & $v$ & $y$ \\
\hline$P_{1}$ & -21 & 0 & $\infty$ & $\infty$ \\
$P_{2}$ & -102 & 0 & 0 & 2 \\
$P_{3}$ & -57 & -540 & -1 & 5 \\
$P_{4}$ & -57 & 540 & 1 & 5 \\
$P_{5}$ & 303 & 4860 & 1 & -5 \\
$P_{6}$ & 123 & 0 & $\infty$ & $\infty$ \\
$P_{7}$ & 303 & -4860 & -1 & -5 \\
\hline
\end{tabular}

Hence in the case $b=-1$ we get the Galois group $C_{4}$ only for $n=-3,7$, which corresponds to the polynomials $x^{4}+3 x^{3}+4 x^{2}+2 x+1$ and $x^{4}-7 x^{3}-$ $6 x^{2}+2 x+1$.

From now on we assume that $E_{b}$ is a quartic number field generated by a root $\varrho$ of $F_{b}$ over $\mathbb{Q}$, and $F_{b}$ has Galois group $D_{4}$. Our construction immediately leads to two independent units of $E_{b}$, namely $\varrho$ itself and the unit $\varepsilon$ of $\Omega_{b}$. We will further restrict our considerations to fields $E_{b}$ of signature $(2,1)$. In that case those two units form a maximal independent set of units 
of $E_{b}$. For the signature $(4,0)$ our efforts to find a third independent unit in parametric form were unsuccessful.

In the remainder of this section we show that $\varrho$ and $\varepsilon$ form a fundamental set of units for the order $\mathbb{Z}[\varrho]$. This also means that they form a fundamental system of units for the field $\mathbb{Q}(\varrho)$ whenever $n^{2}+4 b$ and $4(n-4 b)+1$ are square-free and coprime.

Remark. From $16\left(n^{2}+4 b\right)=(4 n+(16 b-1))(4 n-(16 b-1))+(16 b+1)^{2}$ we conclude that a common factor of $n^{2}+4 b$ and $4(n-4 b)+1$ necessarily divides $(16 b+1)^{2}$.

We use a lower regulator bound of Nakamula [10]. Proposition 3 of his article states that the quotient of the regulators of $E_{b}$ and $\Omega_{b}$ is bounded from below by

$$
L:=\frac{1}{2} \log \left(\sqrt[3]{|4(n-4 b)+1| \frac{\left(n^{2}+4 b\right)^{2}}{4}+\left(\frac{317}{27}\right)^{3}}-\frac{290}{27}\right) .
$$

We need to give a lower estimate for $L$. We start with the radicand of the cubic root. For $n \leq-10$ it is of the form

$$
|n|^{5}(1+\lambda)
$$

with

$$
\lambda> \begin{cases}\frac{15}{4|n|}+\frac{8}{n^{2}}+0.048 & \text { for } b=1 \\ -\frac{17}{4|n|}-\frac{8}{n^{2}}+0.051 & \text { for } b=-1\end{cases}
$$

From this we conclude

$$
L>\frac{1}{2} \log \left(|n|^{5 / 3}\left(1+\frac{\lambda}{3}-\frac{\lambda^{2}}{6}\right)-\frac{290}{27}\right)
$$

resulting in

$$
L>\frac{2}{3} \log |n| .
$$

Next we compute an upper estimate for the regulator $R_{E_{b}}$ of the independent units $\varrho$ and $\varepsilon$. We choose the first two conjugates $\varrho^{(1)}$ and $\varrho^{(2)}$ of $\varrho$ for this purpose, and get

$$
R_{E_{b}}=\left|\operatorname{det}\left(\begin{array}{ll}
\log \left|\varrho^{(1)}\right| & \log \left|\varrho^{(2)}\right| \\
\log |\varepsilon| & \log |\varepsilon|
\end{array}\right)\right|=|\log | \varepsilon|| \mid \log \frac{\left|\varrho^{(1)}\right|}{\left|\varrho^{(2)}\right|} .
$$

We begin by estimating the quotient $\left|\varrho^{(1)} / \varrho^{(2)}\right|$. We have

$$
\frac{\varrho^{(1)}}{\varrho^{(2)}}=\frac{-\varepsilon+\sqrt{\varepsilon^{2}+4 b \varepsilon}}{-\varepsilon-\sqrt{\varepsilon^{2}+4 b \varepsilon}} \text {. }
$$


We easily compute

$$
\mu:=\varepsilon^{2}+4 b \varepsilon=\left(n^{2}-4 b n+2 b-(n-4 b) \sqrt{n^{2}+4 b}\right) / 2 .
$$

One obtains the estimates

$$
L_{\varepsilon}<\varepsilon<U_{\varepsilon}
$$

where

$$
L_{\varepsilon}:=|n|+b /|n|-2 / n^{3}, \quad U_{\varepsilon}:=|n|+b /|n|,
$$

and

$$
L_{\mu}<\sqrt{\mu}<U_{\mu}
$$

where

$$
\begin{aligned}
& L_{\mu}:=|n|+2 b+(b-4) /|n|-2 / n^{2}-(8 b+2) /|n|^{3}-4 b / n^{4}, \\
& U_{\mu}:=|n|+2 b+b /|n|+2 / n^{2} .
\end{aligned}
$$

By considering the cases $b= \pm 1$ separately, one obtains

$$
\left|\frac{\varrho^{(1)}}{\varrho^{(2)}}\right|<C=\frac{|n|+1.11}{0.779}
$$

for $|n| \geq 10$.

If the unit group $U:=\langle-1, \varrho, \varepsilon\rangle$ is a proper subgroup of the full unit group $U_{\mathbb{Z}[\varrho]}$ of $\mathbb{Z}[\varrho]$, then the regulator of $E_{b}$ divided by the regulator of $\Omega_{b}$ is $\leq \log (C) / 2$. Showing $\log (C) / 2<L$ therefore proves that $\varrho, \varepsilon$ are a fundamental set of units for $\mathbb{Z}[\varrho]$. Again, it is easy to see that

is tantamount to

$$
\frac{1}{2} \log \frac{|n|+1.11}{0.779}<\frac{2}{3} \log |n|
$$

$$
\frac{|n|+1.11}{0.779|n|^{4 / 3}}<1
$$

and the latter is satisfied for all $n<-5$.

THEOREM 2.6. If the field $E_{b}=\mathbb{Q}(\varrho)$ is generated by $F_{b}$ with dihedral Galois group, then $\varrho$ and $\varepsilon$ are fundamental units of the order $\mathbb{Z}[\varrho]$. They are even fundamental units of $E_{b}$ when $4(n-4 b)+1$ and $n^{2}+4 b$ are both square-free and coprime.

The estimates above prove the theorem for $n \leq-10$. For larger values of $n$ the proof is by directly calculating the unit group of $E_{b}$ with KANT [2].

3. A parametric family of number fields of degree 4. In this part we consider the parametric family of polynomials of degree 4 defined by $f(x)=x^{4}+a x^{3}-2 x^{2}+(1-a) x+1$. This family arises by the same idea of construction as the families in [10], but there only the cases with Galois group $D_{4}$ are presented. The constructive idea is the assumption that $\varrho, \varrho+1$ and $\varrho-1$ are units of the number fields generated by $x^{4}+a x^{3}+b x^{2}+c x+1$ (with $\varrho$ a zero). In this way one gets three families, two of them are studied in 
[10] $\left(x^{4}+a x^{3}-b x^{2}-a x+1\right.$ with $\left.b \in\{1,3\}\right)$, the third family $f(x)$ is presented here. By straightforward calculation it is easily seen that these polynomials are irreducible and have (for $a \geq 3$ ) four real roots. They generate for $a \in \mathbb{N}, a \geq 3$ number fields $F=\mathbb{Q}[\varrho]$ of signature $(4,0)$ with rank $r_{F}=3$. For $a \in\{ \pm 1,0,2\}$ the number fields have signature $(2,1)$. And for $k \in \mathbb{Z}$ the polynomial $f$ generates the same number field $F$ for $a=k$ and $a=1-k$, hence there is no need to consider $a<-1$.

In the following we therefore only consider the case $a \geq 3$.

REMARK. Examples suggest that for infinitely many $a$ the discriminant $d_{f}=4 a^{6}-12 a^{5}+28 a^{4}-36 a^{3}-56 a^{2}+72 a-283$ of $f$ has no quadratic factors, which implies that the order $\mathbb{Z}[\varrho]$ is maximal, the discriminant $D_{F}$ of $F$ equals $d_{f}$, and the polynomials $f$ generate infinitely many number fields.

THEOREM 3.1. The index of $\mathbb{Z}[\varrho]$ in the maximal order of the number field $F$ generated by $f$ is not divisible by 5 or 13 for all $a \geq 3$.

For $a \equiv 3(\bmod 5)$ (and only for those $a)$ we have $d_{f} \equiv 0(\bmod 25)$ but $d_{f} \not \equiv 0\left(\bmod 5^{3}\right)$. The Dedekind test shows that in this case (and therefore in all cases) the order $\mathbb{Z}[\varrho]$ is already 5 -maximal. Similarly for $a \equiv 7(\bmod 13)$ (and only for those $a$ ) we have $d_{f} \equiv 0\left(\bmod 13^{2}\right)$ but $d_{f} \not \equiv 0\left(\bmod 13^{3}\right)$. Again $\mathbb{Z}[\varrho]$ is already 13-maximal. Thus this order is maximal if the discriminant is divisible by only the quadratic factors 25 and/or 169 .

REMARK. Computations show that for $3 \leq a \leq 2000$ there are only 26 number fields with non-maximal order $\mathbb{Z}[\varrho]: a \in\{80,143,326,380,406,425$, 450, 537, 609, 620, 699, 979, 984, 1044, 1049, 1106, 1138, 1235, 1386, 1498, $1508,1540,1667,1695,1825,1906\}$. These fields are partly described with $k \in \mathbb{N}$ by $a=(3+k \cdot 23) \cdot 23+11$ (we find $a=80,609,1138,1667$ in the set above) where $d_{f}$ is divisible by $23^{2}$, and by $a=(19+k \cdot 23) \cdot 23+13$ (we find $a=450,979,1508)$ where $d_{f}$ is again divisible by $23^{2}$. The discriminant $d_{f}$ is divisible by $29^{2}$ for $a=(4+k \cdot 29) \cdot 29+27$ (we find $a=143,984,1825$ ) or $a=(24+k \cdot 29) \cdot 29+3$ (we find $a=699,1540)$. Or $d_{f}$ is divisible by $31^{2}$ and we have $a=(13+k \cdot 31) \cdot 31+22$ (we find $a=425,1386$ ) or $a=(17+k \cdot 31) \cdot 31+10$ (we find $a=537,1498)$. On the other hand, for $a$ in any of these sets of parametric natural numbers, $d_{f}$ is always divisible by the corresponding square.

THEOREM 3.2. The four zeros of $f$ lie in the following four intervals:

$$
\begin{array}{ll}
\varrho_{1} \in\left[-a-1 / a-1 / a^{2},-a\right], & \varrho_{3} \in\left[1 / a, 1 / a+1 / a^{2}\right], \\
\varrho_{2} \in\left[-1+1 / a^{2},-1+1 / a\right], & \varrho_{4} \in[1-2 / 3 a, 1-1 / 2 a] .
\end{array}
$$

For $a \geq 4$, one shows that $f\left(x_{\min }\right) f\left(x_{\max }\right)<0$, where $\left(x_{\min }, x_{\max }\right) \in$ $\left\{\left(-a-1 / a-1 / a^{2},-a\right),\left(-1+1 / a^{2},-1+1 / a\right),\left(1 / a, 1 / a+1 / a^{2}\right),(1-2 / 3 a, 1-\right.$ $1 / 2 a)\}$. This proves the theorem. 
REMARK. Because $\varrho_{3}<1 / a+1 / a^{2}<1 / 2<1-2 / 3 a<\varrho_{4}$ we get the following inequalities for the zeros of $f$ :

$$
-a-1<\varrho_{1}<-a<-1<\varrho_{2}<0<\varrho_{3}<1 / 2<\varrho_{4}<1 .
$$

ThEOREM 3.3. The polynomial $f$ has Galois group $S_{4}$.

To show this we first look at the cubic resolvent $r_{f}$ of $f$. As in [13], we get $r_{f}(x)=x^{3}+4 x^{2}+a(1-a) x+1$ with discriminant $d\left(r_{f}\right)=d_{f}=$ $-4 \alpha^{3}+16 \alpha^{2}+72 \alpha-283$ with $\alpha=a(1-a)$. The resolvent $r_{f}$ is irreducible and we observe that $d\left(r_{f}\right)>0$ for $a \geq 3$. Moreover the discriminant is not a square in $\mathbb{Q}$ because $y^{2}=d\left(r_{f}\right)$ defines an elliptic curve which has no rational point except $\infty$. This implies that $r_{f}$ has Galois group $S_{4}$ and the theorem follows.

Let $\varrho$ be a zero of $f$. In the number field $\mathbb{Q}(\varrho)$ the element $\varrho$ is obviously a unit. Moreover, by definition of $f$ the elements $\varrho+1, \varrho-1 \in \mathbb{Z}[\varrho]$ are units as well, and $(\varrho+1)^{-1}=\varrho^{3}+(a-1) \varrho^{2}-(a+1) \varrho+2$, and $(\varrho-1)^{-1}=$ $-\varrho\left(\varrho^{2}+(a+1) \varrho+(a-1)\right)$.

Since $\varrho-1$ and $\varrho$ are units, so is their quotient $\vartheta:=(\varrho-1) / \varrho$.

THEOREM 3.4. The three units $\{\varrho, \varrho+1,1-1 / \varrho\}$ form a system of independent units of the order $\mathbb{Z}[\varrho]$. Moreover this set is a fundamental system of units for $a \geq 3$.

To show this, we first assume $(\varrho+1)^{k}= \pm \varrho^{l}$ with $k \in \mathbb{N}, l \in \mathbb{Z}$. This implies that $|\varrho+1|^{k}=|\varrho|^{l}$. Let $k>0$. Because $1<\varrho_{4}+1<2$ and $0<\varrho_{4}<1$ we get $l<0$; from $a-1<\left|\varrho_{1}+1\right|<a<\left|\varrho_{1}\right|<a+1$ we get $l>0$, which yields a contradiction.

The pairwise independence for the other two cases is shown in a similar way with the help of the sequence of inequalities for $\vartheta$ (for $a>3$ ):

$$
2-a<\vartheta_{3}<1-a<-1<-\frac{1}{a}<\vartheta_{4}<-\frac{1}{2 a}<0<1<\vartheta_{1}<\frac{3}{2}<\vartheta_{2}<3 .
$$

Now we assume that $\vartheta^{k}= \pm \varrho^{l}(\varrho+1)^{m}$ where $k, l, m \in \mathbb{Z}$. Without loss of generality let $k>0$. If $l, m>0$ then the image of the canonical embedding $\varphi_{2}$ with $\varrho \mapsto \varrho_{2}$ yields $\left|\vartheta_{2}\right|^{k}=\left|\varrho_{2}\right|^{l}\left|\varrho_{2}+1\right|^{m}$, which is impossible because the left hand side is $>1$ and the right is $<1$. The consideration of the other canonical embeddings $\varphi_{1}, \varphi_{3}$ and $\varphi_{4}$ leads also to contradictions in the remaining cases.

Thus we have shown that the three units $\varrho, \varrho+1$ and $\vartheta$ are a maximally independent set of units of $\mathbb{Q}(\varrho)$.

A lower bound for the regulator $R$ of the unit group of the maximal order of $\mathbb{Q}(\varrho)$ is given in $[12]$ :

$$
R \geq \sqrt{\left(\frac{\left(\log \left(\left|D_{F}\right| / 16\right)\right)^{2}}{20}\right)^{3} \frac{1}{8}}
$$


(In general we have $D_{F}=c^{2} d_{f}$ for some constant $c \in \mathbb{N}$, but in infinitely many cases (see the first Remark of this section) the order $\mathbb{Z}[\varrho]$ seems to be already maximal, so $c=1$ as assumed. The inequality holds in general for $\mathbb{Z}[\varrho]$ with $D_{F}$ replaced by $d_{f}$.)

Since $d_{f}>\frac{64}{17} a^{6}$ for $a \geq 49$ we get the lower bound $R_{\text {low }}$ of the regulator:

$$
\begin{aligned}
\frac{1}{\sqrt{64000}}\left(\log \left(\frac{d_{f}}{16}\right)\right)^{3} & \geq \frac{1}{253}\left(6 \log (a)+\log \left(\frac{4}{17}\right)\right)^{3} \\
& \geq \frac{(6 \log (a)-1.5)^{3}}{253}=: R_{\text {low }}
\end{aligned}
$$

The regulator $R_{\varrho}$ for a system of independent units $\{\varrho, \varrho+1, \vartheta\}$ of $\mathbb{Z}[\varrho]$ is defined by

$$
R_{\varrho}=\left|\operatorname{det}\left(\begin{array}{lll}
\log \left(\left|\varrho_{1}+1\right|\right) & \log \left(\left|\varrho_{1}\right|\right) & \log \left(\left|\vartheta_{1}\right|\right) \\
\log \left(\left|\varrho_{3}+1\right|\right) & \log \left(\left|\varrho_{3}\right|\right) & \log \left(\left|\vartheta_{3}\right|\right) \\
\log \left(\left|\varrho_{4}+1\right|\right) & \log \left(\left|\varrho_{4}\right|\right) & \log \left(\left|\vartheta_{4}\right|\right)
\end{array}\right)\right| .
$$

Computing the determinant and taking into account the size of the arguments of the logarithms, respectively the signs of the values of the logarithms, we can estimate $R_{\varrho}$ from above:

$$
\begin{aligned}
R_{\varrho} \leq & \log \left(\left|\varrho_{1}+1\right|\right) \log \left(\frac{1}{\left|\varrho_{3}\right|}\right) \log \left(\frac{1}{\left|\vartheta_{4}\right|}\right)+\log \left(\left|\varrho_{1}\right|\right) \log \left(\left|\vartheta_{3}\right|\right) \log \left(\left|\varrho_{4}+1\right|\right) \\
& +\log \left(\left|\varrho_{4}+1\right|\right) \log \left(\frac{1}{\left|\varrho_{3}\right|}\right) \log \left(\left|\vartheta_{1}\right|\right)+\log \left(\frac{1}{\left|\varrho_{4}\right|}\right) \log \left(\left|\vartheta_{3}\right|\right) \log \left(\left|\varrho_{1}+1\right|\right) \\
& +\log \left(\frac{1}{\left|\vartheta_{4}\right|}\right) \log \left(\left|\varrho_{3}+1\right|\right) \log \left(\left|\varrho_{1}\right|\right) .
\end{aligned}
$$

Now all factors are positive. Using the approximations of $\varrho$ and $\vartheta$ and the inequalities $\log (2)<0.7, \log (1+1 / a)<0.02$ and $\log \left(1+1 / a+1 / a^{2}\right)<$ $\log \left(1+1 / a+1 / a^{2}+1 / a^{3}\right)<0.021$, one shows that for $a \geq 50$,

$$
R_{\varrho} \leq \log (a)^{3}+1.461 \cdot \log (a)^{2}+0.05822 \cdot \log (a)+0.00042=: R_{\mathrm{up}} .
$$

Finally, we obtain

$$
1<\frac{R}{R_{\text {low }}}<\frac{R_{\text {up }}}{R_{\text {low }}}<2,
$$

where the last inequality holds for $a>44$. This comes from the inequality $\frac{R_{\text {up }}}{R_{\text {low }}}(\log (44))<2$ and because the quotient is decreasing for $a>44$. So the index of the unit system $\{\varrho, \varrho+1, \vartheta\}$ in a fundamental system of units is lower than 2 , which implies that for $a>50$ the units $\{\varrho, \varrho+1,1-1 / \varrho\}$ are fundamental units of $\mathbb{Z}[\varrho]$.

The remaining cases $3 \leq a \leq 44$ are proved by direct calculations with KANT [2]. 
4. A second family of number fields of degree 4. In an analogous way as in Section 3 we show that for the family of polynomials $f_{a}(x)=$ $x^{4}-\left(a^{2}+a+1\right) x^{2}+\left(a^{2}+a\right) x-1$ the set $\{\varrho, \varrho-1, \varrho-a\}$ forms a fundamental system of units of the number field generated by a root of $f_{a}$.

Calculations show that the $f_{a}(x)$ are irreducible and have four real roots for $a \notin\{0, \pm 1,-2\}$. Computations of examples suggest that for $a \in \mathbb{Z}^{\geq 2}$ the $f_{a}$ generate infinitely many number fields of signature $(4,0)$ with unit rank 3 . For $a \in\{0, \pm 1,-2\}$ the number fields have signature $(2,1)$. Moreover $f_{a}$ and $f_{-a-1}$ generate the same number field, hence there is no need to consider $a<-2$.

In the following we therefore only consider the case $a \geq 2$.

The discriminant of $f_{a}$ is $d_{f}=4 a^{10}+20 a^{9}+9 a^{8}-84 a^{7}-74 a^{6}+156 a^{5}+$ $169 a^{4}-60 a^{3}-396 a^{2}-320 a-400$. Computations show that $d_{f} \equiv 0\left(\bmod 2^{4}\right)$ but $d_{f} \not \equiv 0\left(\bmod 2^{5}\right)$ for any $a \in \mathbb{Z}$, and $d_{f} \equiv 0\left(\bmod 5^{2}\right)$ for $a \equiv 0,4(\bmod 5)$ but $d_{f} \not \equiv 0\left(\bmod 5^{3}\right)$ for any $a \equiv 0,4(\bmod 5)$. Using the Dedekind test for the maximality of an order we get:

THEOREM 4.1. The index of $\mathbb{Z}[\varrho]$ in the maximal order of the number field generated by $f_{a}$ is not divisible by 2 or 5 for all $a \geq 2$.

Numerical approximations of the roots of $f_{a}$ lead to:

THEOREM 4.2. The four roots of $f_{a}$ lie in the four intervals:

$$
\begin{array}{ll}
\varrho_{1} \in[-a-2,-a-1], & \varrho_{3} \in\left[1-1 / a^{2}, 1-1 / a^{3}\right], \\
\varrho_{2} \in\left[1 / a^{3}, 1 / a^{2}\right], & \varrho_{4} \in\left[a+1 / a^{4}, a+1 / a^{3}\right] .
\end{array}
$$

As in Section 3 we compute the Galois group of $f_{a}$ with the cubic resolvent $r_{f_{a}}=x^{3}+2\left(a^{2}+a+1\right) x^{2}+\left(\left(a^{2}+a+1\right)^{2}+4\right) x+a^{2}(a+1)^{2}$ to be $S_{4}$. The roots of $f_{a}$ are units and we have:

THEOREM 4.3. The three units $\{\varrho, \varrho-1, \varrho-a\}$ are independent units of the order $\mathbb{Z}[\varrho]$. They form a fundamental system of units for $a \geq 2$.

To prove this theorem the following proposition is helpful:

Proposition 4.4. The three units $\{\varrho, \varrho-1, \varrho-a\}$ are independent if and only if $\{\varrho,(\varrho-1) / \varrho, \varrho(\varrho-a)\}$ are independent.

The independence of $\{\varrho,(\varrho-1) / \varrho, \varrho(\varrho-a)\}$ is proved similarly to Theorem 3.4. The fundamentality of the set of Theorem 4.3 is proved by approximations of the regulator as in 3.4:

$$
R_{\text {low }}=\frac{(10 \log a+\log (1 / 4)+\log (1+5 / a))^{3}}{\sqrt{64000}}
$$

and

$$
R_{\mathrm{up}}=8.07 \log ^{3} a+3 \log ^{2} a,
$$


which implies

$$
\frac{R_{\text {up }}}{R_{\text {low }}}<3
$$

for $a \geq 150$. Finally, we have to show that any unit of the form $\theta=$ $\pm \varrho^{m_{1}}(\varrho-1)^{m_{2}}(\varrho-a)^{m_{3}}$ with $m_{i} \in\{0,1\}$ is not a square in the order $\mathbb{Z}[\varrho]$. For $\left(m_{1}, m_{2}, m_{3}\right) \in\{(1,0,0),(0,1,0),(0,0,1),(1,1,0),(1,0,1),(1,1,1)\}$ there exists for all $a \in \mathbb{Z}$ a negative conjugate of $\theta$, which implies that $\theta$ cannot be a square. In the remaining case $\left(m_{1}, m_{2}, m_{3}\right)=(0,1,1)$ the unit $(\varrho-1)(\varrho-a)=\varrho^{2}-(a+1) \varrho+a$ cannot be a square either for $a \equiv 0$ $(\bmod 2)$ : consider $\alpha \in \mathbb{Z}[\varrho]$ with $\alpha^{2}=\varrho^{2}-(a+1) \varrho+a$; this implies for every choice of $a \in \mathbb{Z}$ a contradiction concerning the coefficients of $\alpha^{2}$ and $\varrho^{2}-(a+1) \varrho+a$ modulo 2 . For $a \not \equiv 1,7(\bmod 8)$ the unit considered cannot be a square either for the same reasons modulo 8. (Even for other choices of the parameter a computations show that $\{\varrho, \varrho-1, \varrho-a\}$ are fundamental.)

Acknowledgments. We would like to thank the referee for insightful comments and suggestions.

\section{References}

[1] G. Frei, Fundamental systems of units in number fields $\mathbb{Q}\left(\sqrt{D^{2}+d}, \sqrt{D^{2}+4 d}\right)$ with $d \mid D$, Arch. Math. (Basel) 36 (1981), 137-144.

[2] Kant, http://www.math.tu-berlin.de/ kant.

[3] S. Katayama, The abc conjecture, fundamental units and the simultaneous Pell equations, Proc. Jangjeon Math. Soc. 1 (2000), 19-26.

[4] O. Lecacheux, Unités de corps de nombres et courbes de genre un et deux, in: K. Dilcher (ed.), Number Theory (Halifax, 1994), CMS Conf. Proc. 15, Amer. Math. Soc., Providence, RI, 1995, 229-243.

[5] - Familles de corps de degré 4 et 8 liées à la courbe modulaire $X_{1}(16)$, in: S. David (ed.), Séminaire de théorie des nombres (Paris, 1991-92), Progr. Math. 116, Birkhäuser, Boston, MA, 1994, 89-105.

[6] F. Leprévost, M. Pohst et A. Schöpp, Familles de polynômes liées aux courbes modulaires $X_{1}(l)$ unicursales et points rationnels non-triviaux de courbes elliptiques quotient, Acta Arith. 110 (2003), 401-410.

[7] G. Lettl and A. Pethö, Complete solution of a family of quartic Thue equations, Abh. Math. Sem. Univ. Hamburg 65 (1995), 365-383.

[8] Magma, http://magma.maths.usyd.edu.au/magma/.

[9] T. Nagell, Zur Arithmetik der Polynome, Abh. Math. Sem. Univ. Hamburg 1 (1922), 179-194.

[10] K. Nakamula, Certain quartic fields with small regulators, J. Number Theory 57 (1996), 1-21.

[11] G. Niklasch and N. P. Smart, Exceptional units in a family of quartic number fields, Math. Comp. 67 (1998), 759-772.

[12] M. E. Pohst and H. Zassenhaus, Algorithmic Algebraic Number Theory, Cambridge Univ. Press, 1989.

[13] J. Rotmann, Galois Theory, Springer, 1990. 
[14] A. Schöpp, Fundamental units in a parametric family of not totally real quintic number fields, J. Théor. Nombres Bordeaux, to appear.

[15] H.-J. Stender, Grundeinheiten für einige unendliche Klassen reiner biquadratischer Zahlkörper mit einer Anwendung auf die diophantische Gleichung $x^{4}-a y^{4}= \pm c$ $(c=1,2,4$ oder 8), J. Reine Angew. Math. 264 (1973), 207-220.

[16] - Eine Formel für Grundeinheiten in reinen algebraischen Zahlkörpern dritten, vierten und sechsten Grades, J. Number Theory 7 (1975), 235-250.

[17] —, "Verstümmelte" Grundeinheiten für biquadratische und bikubische Zahlkörper, Math. Ann. 232 (1978), 55-64.

[18] K. Wang, Fundamental unit systems and class number of real biquadratic number fields, Proc. Japan Acad. Ser. A Math. Sci. 77 (2001), no. 9, 147-150.

[19] L. C. Washington, A family of cyclic quartic fields arising from modular curves, Math. Comp. 57 (1991), 763-775.

\section{LIASIT}

Université du Luxembourg

162 A, Avenue de la Faïencerie

L-1511 Luxembourg

E-mail: Franck.Leprevost@univ.lu
Fakultät II-Mathematik MA 8-1

Technische Universität Berlin

Strasse des 17. Juni 136

D-10623 Berlin, Germany

E-mail:pohst@math.tu-berlin.de schoepp@math.tu-berlin.de

Received on 14.6.2004

and in revised form on 24.1.2007 